\title{
Genome-scale reconstruction and in silico analysis of Klebsiella oxytoca for 2,3-butanediol production
}

\author{
Jong Myoung Park, Hyohak Song ${ }^{*}$, Hee Jong Lee and Doyoung Seung
}

\begin{abstract}
Background: Klebsiella oxytoca, a Gram-negative, rod-shaped, and facultative anaerobic bacterium, is one of the most promising 2,3-butanediol (2,3-BD) producers. In order to improve the metabolic performance of $K$. oxytoca as an efficient biofactory, it is necessary to assess its metabolic characteristics with a system-wide scope, and to optimize the metabolic pathways at a systems level. Provision of the complete genome sequence of $K$. oxytoca enabled the construction of genome-scale metabolic model of $K$. oxytoca and its in silico analyses.

Results: The genome-scale metabolic model of $K$. oxytoca was constructed using the annotated genome with biochemical and physiological information. The stoichiometric model, KoxGSC1457, is composed of 1,457 reactions and 1,099 metabolites. The model was further refined by applying biomass composition equations and comparing in silico results with experimental data based on constraints-based flux analyses. Then, the model was applied to in silico analyses to understand the properties of $K$. oxytoca and also to improve its capabilities for 2,3-BD production according to genetic and environmental perturbations. Firstly, in silico analysis, which tested the effect of augmenting the metabolic flux pool of 2,3-BD precursors, elucidated that increasing the pyruvate pool is primarily important for 2,3-BD synthesis. Secondly, we performed in silico single gene knockout simulation for 2,3-BD overproduction, and investigated the changes of the in silico flux solution space of a IdhA gene knockout mutant in comparison with that of the wild-type strain. Finally, the KoxGSC1457 model was used to optimize the oxygen levels during fermentation for 2,3-BD production.

Conclusions: The genome-scale metabolic model, KoxGSC1457, constructed in this study successfully investigated metabolic characteristics of K. oxytoca at systems level. The KoxGSC1457 model could be employed as an useful tool to analyze its metabolic capabilities, to predict its physiological responses according to environmental and genetic perturbations, and to design metabolic engineering strategies to improve its metabolic performance.
\end{abstract}

\section{Background}

There is growing interest in the production of 2,3butanediol (2,3-BD) by microbial fermentation, as it can be easily converted to methyl ethyl ketone and tetramethyl ether, blending agents for gasoline, and 1,3-butadiene, an intermediate in synthetic rubber manufacture [1-4]. Klebsiella oxytoca is known as one of the most promising 2,3-BD producers [1-10], and its whole genome sequences have been reported recently. The genome of $K$. oxytoca KCTC1686 consists of a chromosome of 5,974,109 bp with a 56.05\% GC content, including 5,488 coding genes [11]. The genome of $K$. oxytoca E718 is composed of a chromosome of 6,097,032 bp and two plasmids of 324,906 bp and

\footnotetext{
* Correspondence: hyohaks@gscaltex.com

Research and Development Center, GS Caltex Corporation, 104-4 Munji-dong, Yuseong-gu, Daejeon 305-380, Republic of Korea
}

110,781 bp with a $55.5 \%$ GC content, including 5,909 coding genes [12]. More recently, we isolated $K$. oxytoca KCTC12133BP from a cattle farm and sequenced its whole genome, but the sequence information, which has not yet been published, consists of a chromosome of $5,903,932$ bp and a plasmid of 109,773 bp with a $55.4 \%$ GC content, including 5,793 coding genes.

The most important characteristics of $K$. oxytoca is to produce large amounts of $\mathrm{C} 3 / \mathrm{C} 4$ diols, 1,3-propanediol (1,3-PD) and 2,3-BD, using various carbon sources [10,13-20]. For example, a lactate deficient mutant of K. oxytoca coproduced 83.6 and $60.1 \mathrm{~g} / \mathrm{L}$ of $1,3-\mathrm{PD}$ and 2,3-BD, respectively, in a fed-batch fermentation utilizing mixed substrates of glycerol and sucrose [13]. K. oxytoca also could produce more than $95 \mathrm{~g} / \mathrm{L}$ of 2,3-BD from glucose with a yield of $0.478 \mathrm{~g} / \mathrm{g}(95.6 \%$ of the theoretical 
maximum yield) and a productivity of $1.71 \mathrm{~g} / \mathrm{L} / \mathrm{h}$ in a batch fermentation, in which the agitation speed was switched from 300 to $200 \mathrm{rpm}$ during the fermentation [10]. The availability of oxygen to $K$. oxytoca significantly affects its physiology and 2,3-BD production [21-23]. Another striking aspect of $K$. oxytoca is to readily metabolize glycerol, an inevitable by-product of biodiesel production, into biomass and products of value [13,24,25].

The aforementioned advantages make $K$. oxytoca an attractive host for industrial applications. Furthermore, the U.S. National Institute of Health (NIH, Guidelines for Research Involving Recombinant DNA Molecules, 2002) has reported that $K$. oxytoca belongs to risk group 1 (RG 1), recognizing it as a GRAS (Generally Regarded As Safe) organism. However, in order to use this organism on an industrial scale, the strain should be further developed. Systems metabolic engineering allows the rational design of metabolic networks for the overproduction of target compounds and the creation of industrially useful microorganisms [26-31]. Here, and in silico genome-scale metabolic model of K. oxytoca, KoxGSC1457, was constructed based on genome information, databases, and experimental data. The KoxGSC1457 model is composed of 1,457 reactions and 1,099 metabolites (Additional file 1 and Additional file 2). The model was carefully examined by in silico analyses for genetic and environmental perturbations. The in silico analysis using the model predicted that the pyruvate pool is mostly important for 2,3-BD synthesis, and this was verified by fermentation of the $l d h A$ gene knockout mutant. Also, the model showed that the availability of oxygen strongly affected the production of 2,3-BD by $K$. oxytoca.

\section{Results and discussion}

Genome-scale reconstruction and general features of $K$. oxytoca metabolic network

The metabolic network of $K$. oxytoca was initially reconstructed based on genome annotation information and metabolic pathway databases [32,33]. The reconstructed metabolic network was further refined by comparing it with experimental data and incorporating biomass formation reactions. The composition of biomass components, including carbohydrates, amino acids, and lipids, was experimentally determined using cells collected in the mid-exponential growth phase (Additional file 1 and Additional file 3). After validation of the genome-scale metabolic model of $K$. oxytoca (KoxGSC1457) was completed, 1,457 metabolic reactions and 1,099 metabolites were included in the final construction (Figure 1 and 2, Table 1 and Additional file 1 and Additional file 2). The KoxGSC1457 model consists of 1,228 intracellular metabolic reactions and 229 transport reactions. 19.57\% of the total open reading frames (ORFs), corresponding to
1,074 genes of 5,488 ORFs, was incorporated into the model (Table 1).

The metabolic reactions in KoxGSC1457 were classified into 8 different subsystems, comprising 97 sub-metabolisms: amino acid metabolism, carbohydrate metabolism, metabolism of cofactors and vitamins, nucleotide metabolism, lipid metabolism, energy metabolism, transporters, and others (Figure 2 and Additional file 1). Figure 2A details the numbers and percentages of reactions associated with genes for each subsystems. Amino acid metabolism ranks as the largest subsystem in KoxGSC1457, followed by carbohydrate metabolism. The sum of the three largest subsystems, amino acid metabolism, carbohydrate metabolism, and metabolism of cofactors and vitamins, account for more than a half of the total number of reactions. The percentage of reactions assigned to ORFs in KoxGSC1457 was 79.7\%. The remaining $20.3 \%$ of reactions included in the model contained lumped multistep reactions, spontaneous reactions, reactions added to fill missing links, or several transport reactions (Table 1). More than $90 \%$ of the reactions in 6 subsystems, except for transporters and energy metabolism are associated with genes (Figure 2A). Figure $2 \mathrm{~B}$ and $\mathrm{C}$ describe the classification of genes and metabolites in KoxGSC1457. The smaller percentages of unique metabolites for each category compared with those of unique genes imply that several metabolites participate with reactions in different subsystems, while most genes function to a specific subsystem (Figure 2B).

The KoxGSC1457 model contains the reactions for 2,3-BD biosynthesis catalyzed by acetolactate synthase (E.C. 2.2.1.6), acetolactate decarboxylase (E.C. 4.1.1.5), and acetoin reductase (E.C. 1.1.1.4). The model also includes a spontaneous reaction that converts $\alpha$-acetolactate into diacetyl $\left(\mathrm{C}_{4} \mathrm{H}_{6} \mathrm{O}_{2}\right)$ and $\mathrm{CO}_{2}$ in the presence of oxygen. Diacetyl can then be catalyzed into acetoin $\left(\mathrm{C}_{4} \mathrm{H}_{8} \mathrm{O}_{2}\right)$, which is the precursor of 2,3-BD, by diacetyl reductase (E.C. 1.1.1.303). K. oxytoca has its specialty for glycerol utilization by oxidative and reductive routes. Thus, the model encompasses the reactions for glycerol utilization, which are catalyzed by glycerol kinase (E.C. 2.7.1.30) and glycerol-3-phosphate dehydrogenase (E.C. 1.1.5.3) of the oxidative route, and NAD-dependent glycerol dehydrogenase (E.C. 1.1.1.6) and dihydroxyacetone kinase (E.C. 2.7.1.29) of the reductive route. Finally, the refined KoxGSC1457 model was used to understand the metabolic characteristics of $K$ oxytoca and was applied to analyze the strategies for $2,3-\mathrm{BD}$ production.

\section{Effects of increasing the metabolic flux pools of 2,3-BD precursors in $K$. oxytoca}

$K$. oxytoca is naturally capable of producing 2,3-BD by metabolizing various carbon sources such as lactose, galactose, glucose, xylose, and glycerol [9,10,13-16,18]. 2,3- 


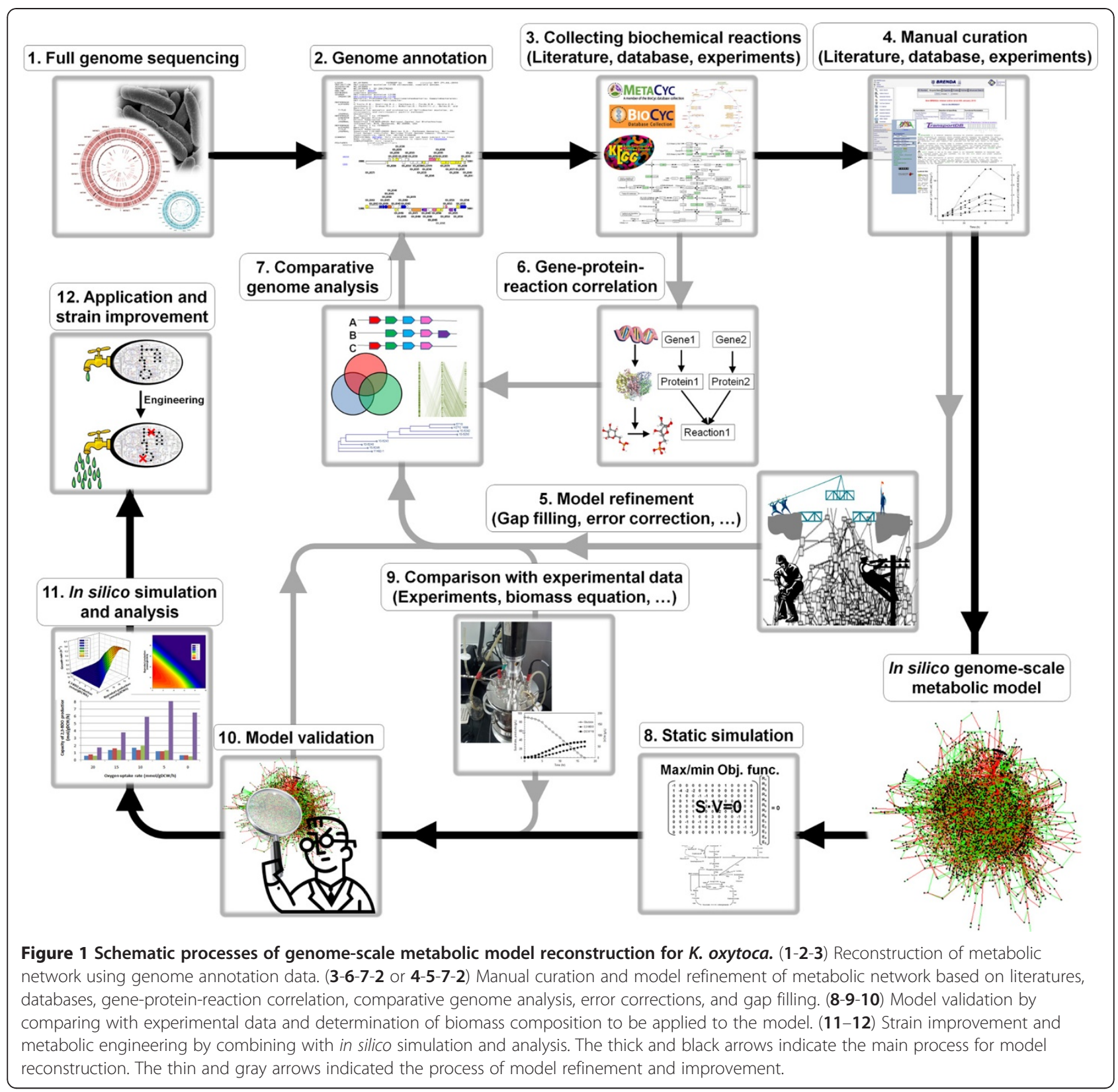

BD is synthesized from pyruvate by enzymes encoded by the $\operatorname{budB}, \operatorname{budA}$, and budC genes in a operon $[2,34]$. Two pyruvates from glycolysis are converted to 2-acetolactate by releasing $\mathrm{CO}_{2}$ (budB). 2-acetolactate is then transformed into acetoin by decarboxylation to $\mathrm{CO}_{2}$ (budA). 2,3-BD is then synthesized from acetoin with oxidation of $\mathrm{NADH}$ into $\mathrm{NAD}^{+}$by acetoin reductase (budC) (Figure 3 ). For the synthesis of one mole of 2,3-BD from pyruvate, two moles of $\mathrm{CO}_{2}$ are released and one mole of NADH is oxidized. The respective reactions for 2,3-BD synthesis are reflected in the KoxGSC1457 model. The model was then employed to design the strategy of 2,3-BD overproduction and to understand its metabolic characteristics.
Firstly, the flux solution space of the wild-type strain of $K$. oxytoca correlated with cell growth, 2,3-BD production, and byproducts formation rates were analyzed in order to understand the current state of the wile-type by constraints-based flux analysis simulation using KoxGSC1457 (Figure 3A). As the first priority of cells is survival, the maximal point of an in silico cell growth rate is generally used to predict the actual state of a cell [35-37], which is indicated by an arrow in Figure 3. Byproducts include ethanol and several organic acids, such as lactic acid, acetic acid, succinic acid, and formic acid. The model predicted that the wild-type strain of $K$. oxytoca produces 2,3-BD along with large amounts of 


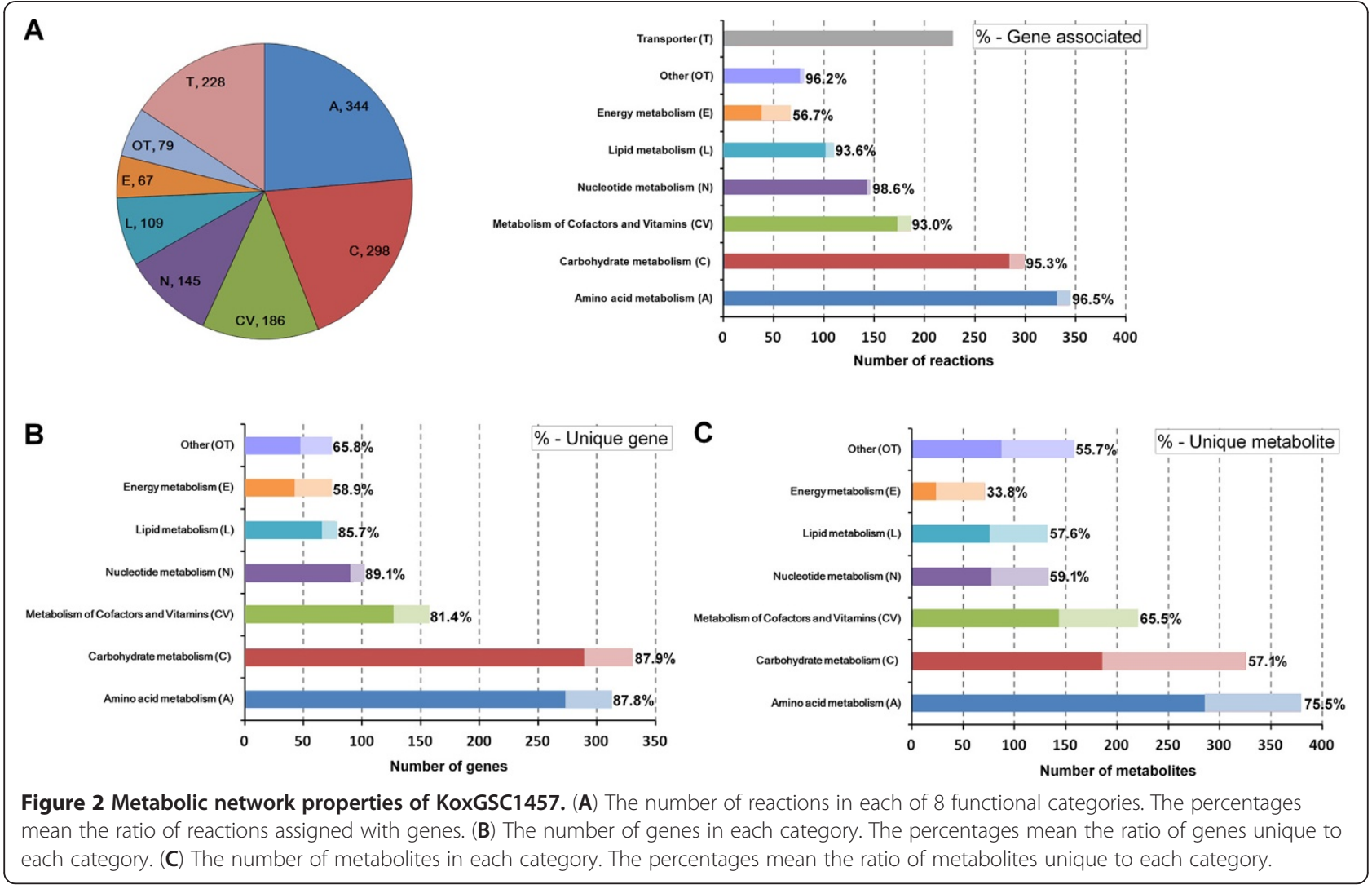

byproducts. This prediction was well supported by the previous studies that the wild-type strains yielded not only 2,3-BD but also lactic acid, formic acid, and ethanol as fermentation end-products (Figure 4C) $[9,10,19]$. Thus, genetic manipulations like gene knockout have

\section{Table 1 Features of the in silico genome-scale metabolic} model of $K$. oxytoca

\begin{tabular}{lc}
\hline Features & Number \\
\hline $\begin{array}{l}\text { Genome feature } \\
\text { Genome size (base pairs, bp) }\end{array}$ & \\
$\quad$ Chromosome 1 & \\
No. of open reading frames (ORFs) & 5,488 \\
$\quad$ Chromosome 1 & \\
In silico metabolic model & \\
No. of reactions (redundant) included in the model & 1,457 \\
$\quad$ No. of biochemical reactions & 1,228 \\
$\quad$ No. of transport reactions & 229 \\
No. of reactions (unique) included in the model & 1,437 \\
No. of metabolites & 1,099 \\
No. of ORFs assigned in metabolic network & 1,074 \\
ORF coverage ${ }^{\text {b }}$ (\%) & 19.57 \\
\hline
\end{tabular}

${ }^{\mathrm{a}}$ The parenthesis includes the value of $\mathrm{G}+\mathrm{C}$ content (\%).

${ }^{\mathrm{b}}$ The number of ORFs participated in the KoxGSC1457 model divided by the total number of ORFs in the $K$. oxytoca genome. been attempted to prevent the formation of byproducts in order to enhance 2,3-BD production $[9,13]$.

Likewise, the KoxGSC1457 model was used to examine the importance of 2,3-BD precursors, and it gave better insights into augmenting the metabolic flux pools of 2,3-BD precursors for 2,3-BD overproduction (Figure 3B and $\mathrm{C}$ ). In order to increase the metabolic flux pools of 2,3-BD precursors as well as to investigate its effects on 2,3-BD overproduction, all reactions participating in the consumption of target precursors were eliminated except for reactions involved in 2,3-BD biosynthetic pathways and essential reactions required for cell growth on the basis of the wild-type strain of KoxGSC1467, as described in Figure 3B. Accordingly, the flux solution spaces were scanned by increasing the flux pool of each precursor (Figure 3C). As a result, increasing the pools of most precursors had no effects on the production of 2,3-BD compared to that of the wild-type strain, as determined through comparison of the flux solution spaces. The model predicted that the surplus fluxes from artificially increased flux pools of most 2,3-BD precursors were not redirected to 2,3-BD synthesis but went toward other pathways for survival related with biomass synthesis, such as amino acids, nucleotides, lipids, and cofactors, prior to arriving at 2,3-BD biosynthetic reactions. In some cases, artificially increasing the flux pool of a 2,3-BD precursor (e.g. phosphoenolpyruvate) had negative influences on 


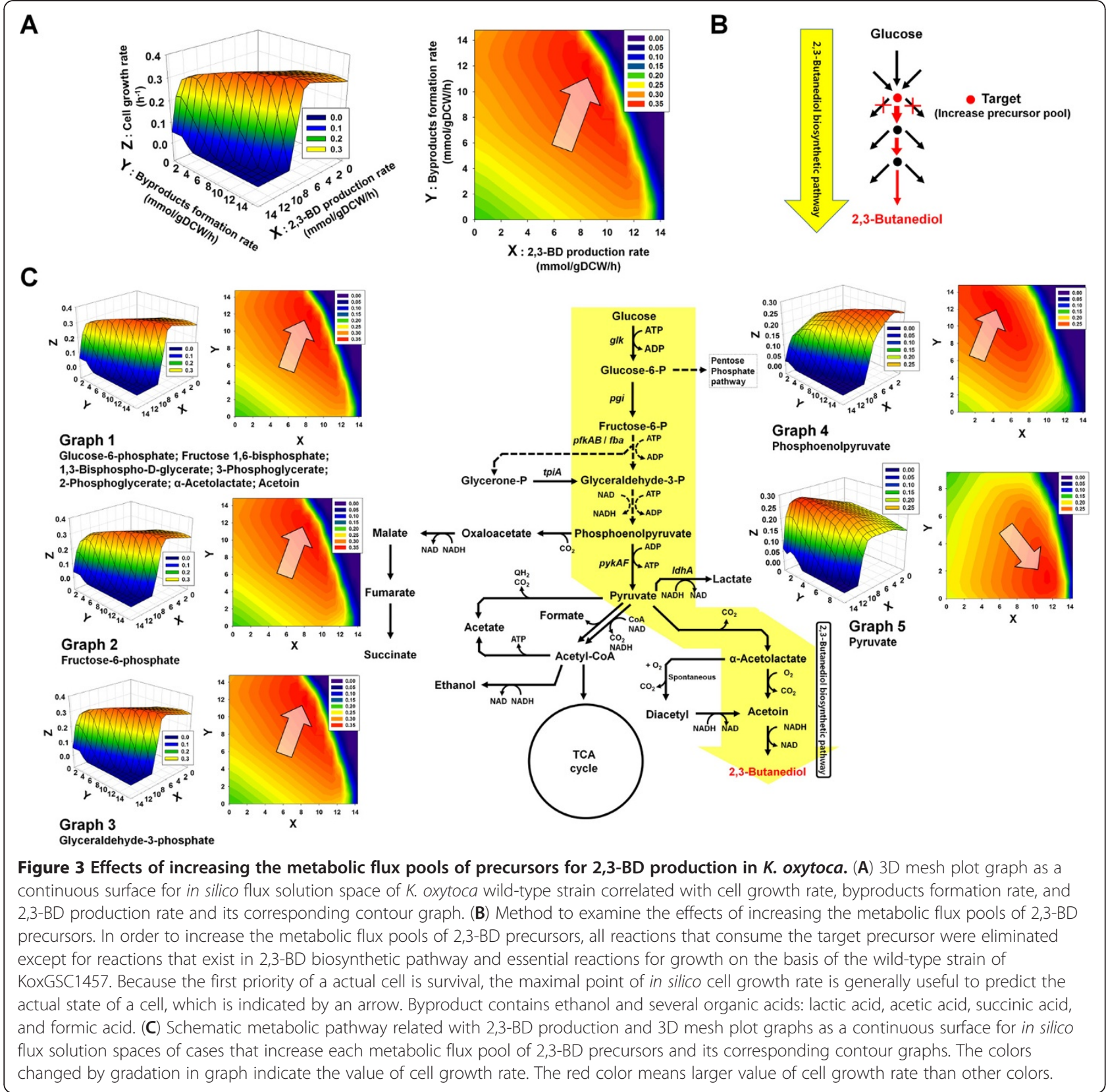

both cell growth and 2,3-BD synthesis by unbalancing the precursor requirements for biomass synthesis. However, if the pyruvate pool was augmented sufficiently, the flux solution space was altered so that KoxGSC1457 was enabled to overproduce 2,3-BD by lowering byproducts formation rates at an optimal cell growth rate (Figure $3 \mathrm{C}$ ). The surplus of the pyruvate pool was sufficiently redirected to 2,3-BD synthesis, resulting in an approximate doubling of the 2,3-BD production rate. Correspondingly, byproducts formation rates decreased dramatically compared with those of the wild-type strain, and consequently, augmentation of the pyruvate pool is one of most important keys for 2,3-BD overproduction.
In silico single gene knockout simulation and changes of flux solution space by IdhA gene knockout in $K$. oxytoca

During fermentation by the $K$. oxytoca wild-type strain, several byproducts, including lactic acid, ethanol, and formic acid were produced (Figure 4). For the overproduction of 2,3-BD in $K$. oxytoca by reducing the formation of byproducts and redirecting the remaining metabolic fluxes towards the synthesis of 2,3-BD, in silico single gene knockout simulation was performed using flux balance analysis (FBA). As a result, the knockout of $l d h A$ gene encoding lactate dehydrogenase, which converts pyruvate into lactic acid with $\mathrm{NADH}$ oxidation, was targeted with top priority for a single gene knockout strategy based on 
A

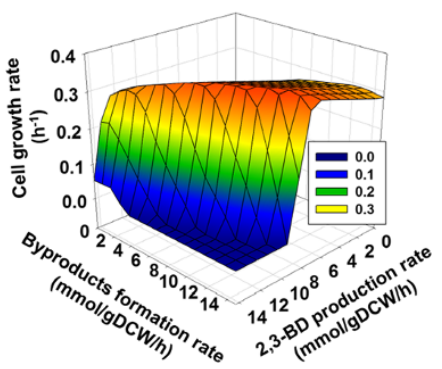

B

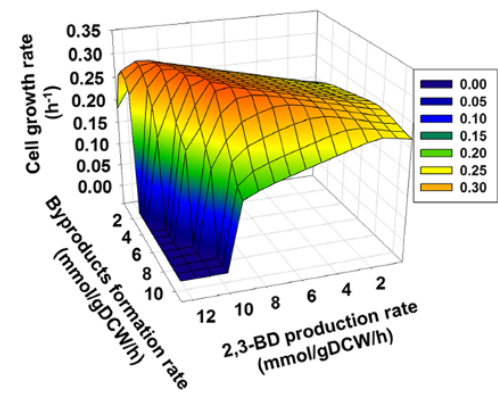

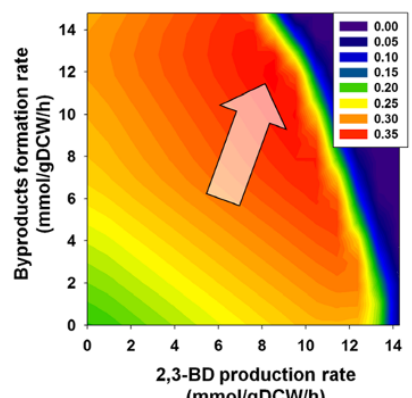

(mmol/gDCW/h)

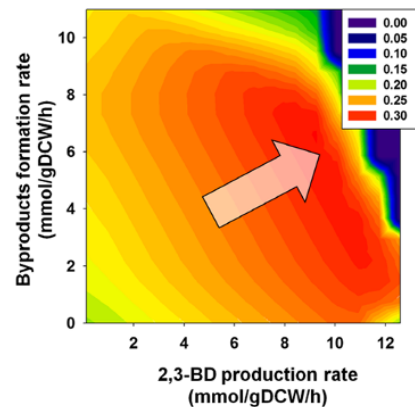

(mmol/gDCW/h)

C

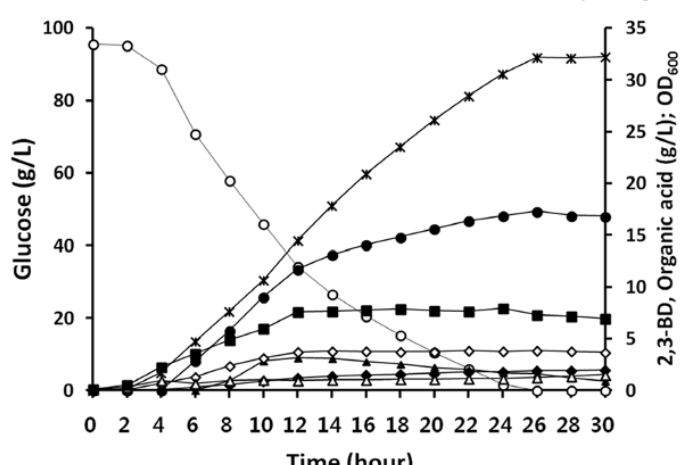

D

Time (hour)

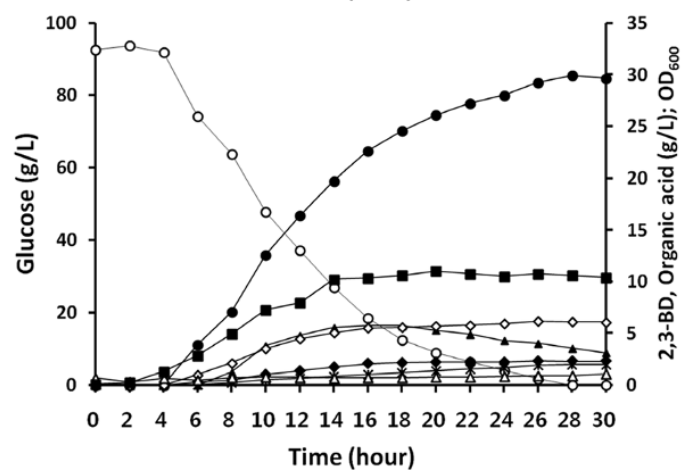

Figure 4 Flux solution spaces and batch fermentation results of wild-type and mutant $(\Delta / d h A)$ strains of $\boldsymbol{K}$. oxytoca. (A) $3 \mathrm{D}$ mesh plot graph as a continuous surface for in silico flux solution space of $K$. oxytoca wild-type strain correlated with cell growth rate, byproducts formation rate, and 2,3-BD production rate and its corresponding contour graph. (B) 3D mesh plot graph as a continuous surface for in silico flux solution space of IdhA gene knockout mutant of $K$. oxytoca correlated with cell growth rate, byproducts formation rate, and 2,3-BD production rate and its corresponding contour graph. The colors changed by gradation in graph indicate the value of cell growth rate. The red color means larger value of cell growth rate than other colors. (C) Batch fermentation result of $K$. oxytoca wild-type strain. (D) Batch fermentation result of IdhA gene knockout mutant of $K$. oxytoca. The arrow indicates the position of in silico optimal cell growth rate. Based on the flux solution space of wild-type strain, the large amount of byproducts were produced during 2,3-BD production. After in silico knockout of IdhA gene, byproducts formation rate was decreased, while 2,3-BD production rate was increased in comparison with wild-type strain. The fermentation was performed at $150 \mathrm{rpm}$, $\mathrm{pH} 6.5$, and $37^{\circ} \mathrm{C}$ in $3 \mathrm{~L}$ working volume. The symbols in fermentation profiles indicates the concentration of glucose $(\mathrm{O}), 2,3-\mathrm{BD}^{(\bullet),} \mathrm{OD}_{600}(\mathbf{\bullet})$, ethanol $(\boldsymbol{\Delta})$, succinic acid $(\bullet)$, lactic acid $(*)$, formic acid $(\diamond)$, and acetic acid $(\Delta)$. 
the criteria of the maximization of 2,3-BD production rate and minimization of byproducts formation rates. Then, the KoxGSC1457 model investigated the metabolic characteristics of the $l d h A$ gene knockout mutant by examining the changes of flux solution space compared with those of the wild-type strain (Figure 4A and B). In the wild-type strain, the model predicted that $K$. oxytoca produces large amounts of byproducts along with 2,3-BD. In the $l d h A$ gene knockout mutant, the optimal point shifted to an increase of 2,3-BD production but to a decreased formation of byproducts, including lactic acid. ldhA gene knockout significantly increased the pyruvate pool that redirected the metabolic flux to 2,3-BD synthesis. This prediction was validated by batch fermentations of $K$. oxytoca wild-type and $l d h A$ gene knockout strains (Figure $4 \mathrm{C}$ and D). K. oxytoca wild-type strain produced $32 \mathrm{~g} / \mathrm{L}$ of lactic acid and $17 \mathrm{~g} / \mathrm{L}$ of $2,3-\mathrm{BD}$ by consuming $90 \mathrm{~g} / \mathrm{L}$ of glucose. However, the $l d h A$ gene knockout mutant dramatically decreased lactic acid production to $1.9 \mathrm{~g} / \mathrm{L}$, which is about $6 \%$ of that produced by the wildtype strain (Figure 4D). Accordingly, 2,3-BD production in the mutant was increased to about $30 \mathrm{~g} / \mathrm{L}$, which is about $176 \%$ of that produced by the wild-type strain. However, cell growth and glucose uptake rates of the $l d h A$ gene knockout mutant were maintained in comparison with those of the wild-type strain because the redox balance of $\mathrm{NAD}^{+} / \mathrm{NADH}$ was not disrupted (Figure $4 \mathrm{C}$ and D). The deletion of lactate dehydrogenase forming lactic acid with the oxidation of NADH seemed to be compensated with 2,3-BD dehydrogenase, producing 2,3-BD with the oxidation of NADH as well, which made 2,3-BD production increase without any retardation of growth. When we compared the in silico flux solution space of the $l d h A$ gene knockout mutant in Figure $4 \mathrm{~B}$ with that of the increasing highly pyruvate pool in Figure $3 \mathrm{C}$, more improvements for 2,3-BD overproduction could be made by further genetic manipulation. In particular, the fermentation results of the $l d h A$ gene knockout mutant in Figure 4D suggests the construction of a mutant preventing the formation of ethanol, formic acid, and succinic acid. For this, the KoxGSC1457 model will be applicable to identify suitable candidates for further genetic manipulations.

\section{Effects of varying oxygen uptake rate for 2,3-BD production in $K$. oxytoca}

For 2,3-BD production by $K$. oxytoca, the dissolved oxygen (DO) level in medium is known as one of the most important environmental factors [21-23]. In order to gain an insight into the effect of oxygen uptake rate on 2,3-BD production in $K$. oxytoca, the oxygen uptake rate was perturbed by constraints-based flux analysis between 0 and $30 \mathrm{mmol} / \mathrm{gDCW}$ (gram dry cell weight)/h and glucose uptake rate was fixed to $10 \mathrm{mmol} / \mathrm{gDCW} / \mathrm{h}$, with maximization and minimization of $2,3-\mathrm{BD}$ production rate as the objective function (Figure 5A). As shown in Figure 5A, the predicted 2,3-BD production rate increased as the oxygen uptake rate was raised to 5 and $10 \mathrm{mmol} / \mathrm{gDCW} / \mathrm{h}$. On the other hand, the 2,3-BD production rate gradually decreased with further increasing oxygen uptake rate (Figure 5A).

This simulation was validated by batch fermentation experiments under different agitation speeds ( $\mathrm{rpm}$ ) and DO levels (Figure 5B and $\mathrm{C}$ ). The agitation speeds were a150, 250, 350, 450, 550, 650, and $750 \mathrm{rpm}$. DO levels were maintained at $5 \%$ and $10 \%$ by automatically adjusting the agitation speed between 50 and 1,000 rpm. The aeration rate was set at $1 \mathrm{vvm}$ (air volume. working volume $^{-1}$. minute ${ }^{-1}$ ) during the whole period of fermentation. The fermentations were performed by using the ldhA gene knockout mutant of $K$. oxytoca, as the most productive 2,3-BD producer up to this point. As a result, the final 2,3-BD concentration $(\mathrm{g} / \mathrm{L})$ and yield $(\mathrm{g} / \mathrm{g}$ glucose) increased through increasing the agitation speed to $350 \mathrm{rpm}$, but further increasing agitation speed caused a decrease of the final 2,3-BD concentration and yield. Meanwhile, the maximal value of 2,3-BD productivity of $2.1 \mathrm{~g} / \mathrm{L} / \mathrm{h}$ was observed at $450 \mathrm{rpm}$. The highest glucose consumption was observed at $550 \mathrm{rpm}$, however, most consumption of carbon source was used to synthesize not 2,3-BD but biomass at $550 \mathrm{rpm}$ and agitation speeds greater than $550 \mathrm{rpm}$. In addition, fermentation at $10 \%$ DO level showed less 2,3-BD concentration, yield, and productivity than at $5 \% \mathrm{DO}$. These results are a strong indication that excessive oxygen exposure during fermentation impairs 2,3-BD overproduction. Since the optimal oxygen level exists beyond the scope of DO as represented in Figure 5, it is recommended to adjust the agitation speed depending on cellular status for 2,3-BD overproduction. These observations were also supported by a previous study, in which the optimal agitation speed existed on 2,3-BD production [10].

\section{Conclusions}

$K$. oxytoca is a promising microorganism which has great potential in the industrial production of 2,3-BD. To make the better use of this organism and to improve industrial applicability, we constructed a genome-scale metabolic model of $K$. oxytoca, KoxGSC1457, consisting of 1,457 reactions and 1,099 metabolites based on information obtained from genome annotation data, database, literature and validation experiments. Using the KoxGSC1457 model, in silico metabolic fluxes were analyzed to investigate the effects of environmental and genetic variation. First, the effects of increasing the metabolic flux pools of 2,3-BD precursors were examined to understand the most important precursor to be augmented for 2,3-BD overproduction. In silico single gene knockout simulation was then performed to identify gene 


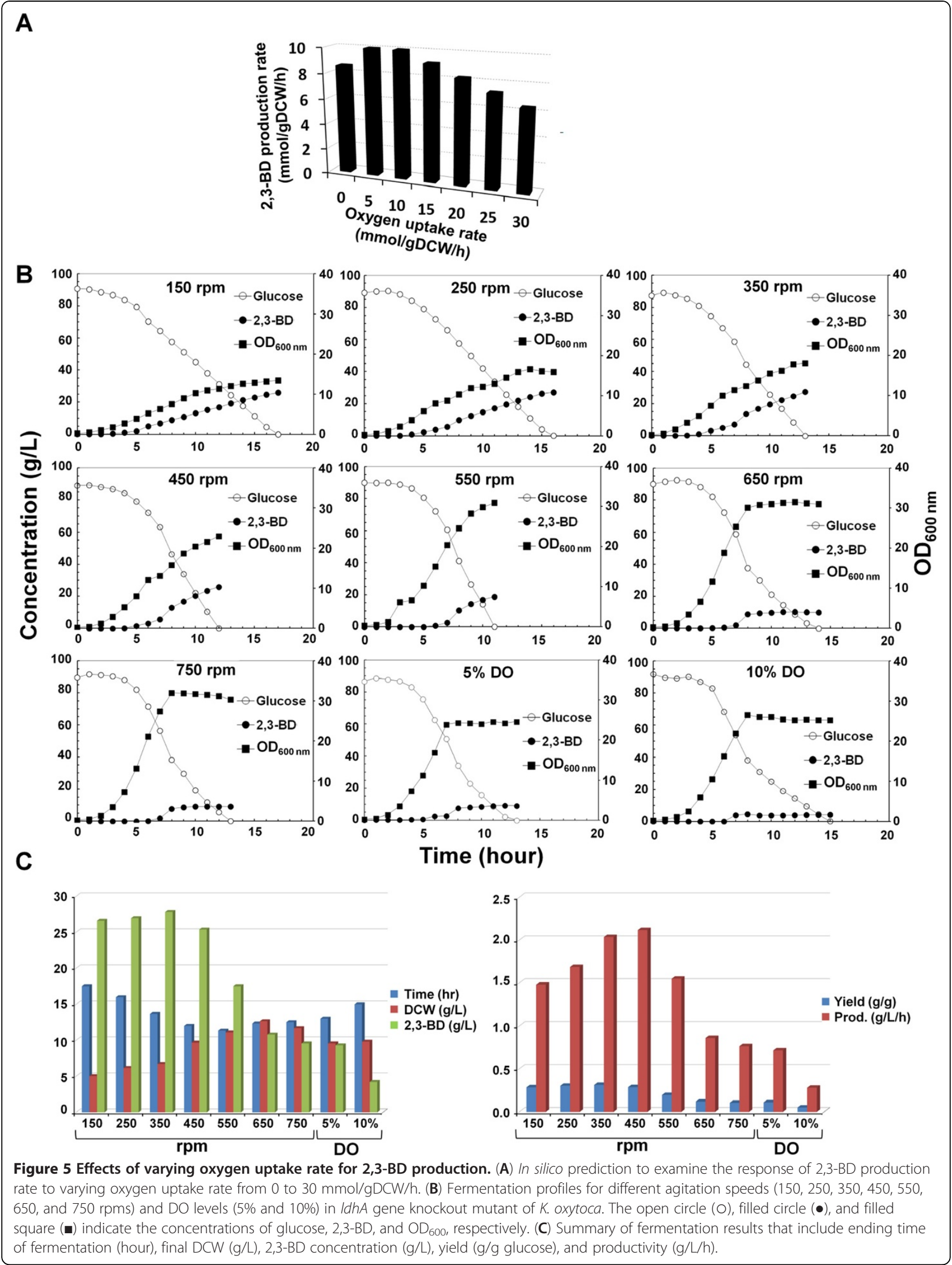


knockout candidates for the reduction of byproduct formation, and the change of flux solution space was then analyzed by elimination of $l d h A$ gene resulting in the $K$. oxytoca ldhA gene knockout mutant, which is one of organisms that shows best performance for 2,3-BD production so far. Finally, to design strategies for better 2,3-BD overproduction, in silico metabolic flux analyses of KoxGSC1457 were executed to examine the effects of varying oxygen uptake rate during 2,3-BD production, and to determine the optimal agitation speed. Through the successful use of the KoxGSC1457 genome-scale metabolic model like those, it gave us the confidence that the model was thoroughly validated in comparison with various experimental data. There are still many difficulties associated with the overall economics of producing 2,3-BD through $K$. oxytoca on an industrial scale. Hence, it is expected that the model constructed in this study will support us to understand cellular physiology on systems level, broaden our insight on this organism, and conquer problems more systematically.

\section{Methods}

Reconstruction of genome-scale metabolic model

Physiological data and biochemical reactions of $K$. oxytoca were collected from various sources, such as experiments, literatures, and public databases, in order to reconstruct the genome-scale metabolic model of $K$. oxytoca. First, information of genome annotation was gained from genome data in NCBI (http://www.ncbi. nlm.nih.gov/). Then, the biochemical reactions of $K$. oxytoca were assembled from various literatures, experiments, and public databases, including the Kyoto Encyclopedia of Genes and Genomes (KEGG, [32]), Biocyc [33], Metacyc [33], TCDB [38], and TransportDB [39]. Second, the draft version of the metabolic model was manually curated and refined based on experimentation, literature, databases, gene-protein-reaction correlations, comparative genome analysis, error corrections, and gap filling. Third, the refined model was validated through comparison with experimental data, including fermentation data. The biomass composition data of $K$. oxytoca were then analyzed and applied to the model. Finally, the validated model was used to analyze the physiology of $K$. oxytoca and to apply to in silico simulations for strain improvement and fermentation optimization (Figure 1).

\section{Biomass composition}

The composition of biomass components was experimentally measured to construct the biomass formation reactions of $K$. oxytoca more accurately. The cells of $K$. oxytoca were cultured in minimal culture medium containing no yeast extract, and the samples were collected in the mid-exponential growth phase. The carbohydrate compositions were determined for neutral and amino sugars (Korea Basic Science Institute, Daejeon, Korea). The neutral and amino sugars were extracted from cells by treating them with $2 \mathrm{M}$ trifluoroacetic acid and $6 \mathrm{~N}$ $\mathrm{HCl}$, respectively, at $100^{\circ} \mathrm{C}$ for 4 hours. The carbohydrates were analyzed by ICD-5000 (Dionex, Sunnyvale, CA, USA) equipped with a CarboPac PA10 column (4.5 $\times$ $250 \mathrm{~mm}$, Dionex) and a CarboPac PA10 cartridge $(4 \times$ $50 \mathrm{~mm}) .16 \mathrm{mM} \mathrm{NaOH}$ was used as a mobile phase with a flow rate of $1.0 \mathrm{~mL} \cdot \mathrm{min}^{-1}$. Then, data were analyzed by Chromelon ver 6.8 software. Amino acid compositions were analyzed by a Hewlett Packard 1100 series HPLC systems equipped with Waters Nova-Pak C18 4 um column $(3.9 \times 300 \mathrm{~mm})$ (Korea Basic Science Institute, Daejeon, Korea). The fatty acid composition of $K$. oxytoca was determined by Sherlock microbial identification system of Sherlock version 6.1 (Korea Research Institute of Bioscience and Biotechnology, Daejeon, Korea). DNA and RNA compositions were analyzed based on genome information of $K$. oxytoca. The DNA composition was calculated by using the GC content $(53 \%)$ of $K$. oxytoca. The RNA composition was calculated based on nucleotide sequences for rRNA and tRNA derived from genome information. The compositions for other components were obtained from literature or assumed reasonably described in Additional file 3.

\section{Constraints-based flux analysis}

In order to perform in silico simulations, and to predict the metabolic characteristics of $K$. oxytoca, constraintsbased flux analysis, including FBA, was carried out under the assumption of a pseudo-steady state [35,40-43]. In order to simulate the in silico model more accurately, the limits of uptake and secretion rates for some metabolites, including amino acids and organic acids such as acetic acid, ethanol, formic acid, lactic acid, pyruvic acid, and succinic acid, were constrained by experimentally measured flux values. Likewise, the limits related with secretion for some metabolites, which were not produced during fermentation such amino acids, were constrained to zero.

The effect of 2,3-BD production rate in response to varying oxygen uptake rate was examined by flux response analysis [44-46]. The 2,3-BD production rate, as objective function, was maximized and minimized according to the changes of oxygen uptake rate from 0 to $30 \mathrm{mmol} /$ $\mathrm{gDCW} / \mathrm{h}$ with a $10 \mathrm{mmol} / \mathrm{gDCW} / \mathrm{h}$ glucose uptake rate.

\section{Fermentation}

For seed preparation, suspended cells from single colonies on Luria-Bertani (LB) agar (Difco Laboratories, Detroit, MI) plates were precultured in $20 \mathrm{~mL}$ test tubes containing $5 \mathrm{~mL}$ culture medium at $37^{\circ} \mathrm{C}$ for $5 \mathrm{~h} .1 \mathrm{~mL}$ aliquots of the preculture were then transferred to $500 \mathrm{~mL}$ 
Erlenmeyer flasks containing $300 \mathrm{~mL}$ culture medium, and the cells were cultivated to an optical density of 1.5 2.0 at $600 \mathrm{~nm}\left(\mathrm{OD}_{600}\right)$. The tube and flask cultivations were placed in a rotary shaker at $150 \mathrm{rpm}$ and $37^{\circ} \mathrm{C}$ (JEIO Tech. Co. SI-900R). The culture medium contained per liter: yeast extract (Becton Dickinson, Le Pont de Claix, France), $5 \mathrm{~g} ; \mathrm{FeSO}_{4} \cdot 7 \mathrm{H}_{2} \mathrm{O}, 0.05 \mathrm{~g} ; \mathrm{ZnSO}_{4} \cdot 7 \mathrm{H}_{2} \mathrm{O}, 0.001 \mathrm{~g}$, $\mathrm{MnSO}_{4} \cdot \mathrm{H}_{2} \mathrm{O}, 0.001$ g; $\mathrm{CaCl}_{2} \cdot 2 \mathrm{H}_{2} \mathrm{O}, 0.001$ g; $\mathrm{MgSO}_{4}$. $7 \mathrm{H}_{2} \mathrm{O}, 0.25 \mathrm{~g}$; $\left(\mathrm{NH}_{4}\right)_{2} \mathrm{SO}_{4}, 6.6 \mathrm{~g} ; \mathrm{K}_{2} \mathrm{HPO}_{4}, 8.7 \mathrm{~g} ; \mathrm{KH}_{2} \mathrm{PO}_{4}$, $6.8 \mathrm{~g}$; trace metal solution, $10 \mathrm{~mL}$. The trace metal solution contains per liter: $\mathrm{FeSO}_{4} \cdot 7 \mathrm{H}_{2} \mathrm{O}, 5 \mathrm{~g}$; $\mathrm{ZnSO}_{4} \cdot 7 \mathrm{H}_{2} \mathrm{O}$, $0.1 \mathrm{~g} ; \mathrm{MnSO}_{4} \cdot \mathrm{H}_{2} \mathrm{O}, 0.1 \mathrm{~g} ; \mathrm{CaCl}_{2} \cdot 2 \mathrm{H}_{2} \mathrm{O}, 0.1 \mathrm{~g} ; \mathrm{HCl} 10 \mathrm{~mL}$.

Batch fermentations were performed in a 5 -L BIOFLO \& CELLIGEN 310 bioreactor (New Brunswick. Scientific Co., Edison, NJ) with $3 \mathrm{~L}$ culture medium containing $90 \mathrm{~g} / \mathrm{L}$ of D-glucose and $300 \mathrm{~mL}$ seed culture. The fermenter was continuously aerated through a $0.2 \mu \mathrm{m}$ membrane filter at a flow rate of $1 \mathrm{vvm}$ (air volume. working volume $^{-1}$. minute $\left.{ }^{-1}\right)$. The temperature was maintained at $37^{\circ} \mathrm{C}$. The $\mathrm{pH}$ was controlled at $6.5 \pm 0.1$ by the automatic feeding of $5 \mathrm{~N} \mathrm{NaOH}$. Foaming was controlled by the addition of Antifoam 289 (Sigma, St. Louis, MO). For biomass composition analysis, the cells were cultured in a medium in which only yeast extract was removed from the culture medium. All fermentations were performed at least three times independently, and the representative results are shown in Figures.

\section{Analytical procedures}

The concentrations of D-glucose and metabolites, including 2,3-BD, formic acid, ethanol, acetic acid, lactic acid, succinic acid, and acetoin, were determined by a highperformance liquid chromatography equipped with UV/ VIS and RI detectors (Agilent 1260 series, Agilent Technologies, Waldbronn, Germany). An Aminiex HPX-87H column (300 mm $\times 7.8 \mathrm{~mm}$, Bio-Rad, Hercules, CA) was isocratically eluted with $0.01 \mathrm{~N} \mathrm{H}_{2} \mathrm{SO}_{4}$ at $80^{\circ} \mathrm{C}$ and a flow rate of $0.6 \mathrm{~mL} / \mathrm{min}$. The $\mathrm{OD}_{600}$ was measured using a UV-vis spectrophotometry (DR5000, Hach Company, $\mathrm{CO})$ to monitor cell growth. Cell concentration, DCW per liter of culture broth, was calculated from the predetermined standard curve relating $\mathrm{OD}_{600}$ to $\mathrm{DCW}$ $\left(1 \mathrm{OD}_{600}=0.3877 \pm 0.0136 \mathrm{~g} \mathrm{DCW} \cdot \mathrm{L}^{-1}\right)$. DCW was determined by filtering the culture broth through a $0.45 \mu \mathrm{m}$ membrane and washing it twice with an equal volume of deionized distilled water. The filtered cells were then dried at $80 \pm 5^{\circ} \mathrm{C}$ overnight and cooled to room temperature in a desiccator prior to weighting.

\section{Additional files}

Additional file 1: List of metabolic reactions in the genome-scale metabolic model of Klebsiella oxytoca.
Additional file 2: List of metabolites in the genome-scale metabolic model of Klebsiella oxytoca.

Additional file 3: Biomass composition of Klebsiella oxytoca.

\section{Abbreviations}

1,3-PD: 1,3-propanediol; 2,3-BD: 2,3-butanediol; ORF: Open reading frame; FBA: Flux balance analysis; KEGG: Kyoto encyclopedia of genes and genomes; LB: Luria-bertani; vvm: Air volume working volume ${ }^{-1} \cdot$ minute $^{-1}$; gDCW: Gram dry cell weight; DO: Dissolved oxygen; rpm: Revolutions per minute.

\section{Competing interests}

The authors declare that they have no competing interests.

\section{Authors' contributions}

$J M P, H S, H J L$, and DS generated ideas. JMP and HS designed the research. JMP performed the research. JMP performed analytical experiments. JMP analyzed data. JMP, HS, HJL, and DS wrote the paper. All authors read and approved the final manuscript.

\section{Acknowledgements}

This work was supported by the Industrial Strategic Technology Development Program (No. 10035241) funded by the Ministry of Knowledge Economy (MKE, Korea).

Received: 4 January 2013 Accepted: 17 February 2013 Published: 23 February 2013

\section{References}

1. Volch M, Jansen NB, Ladisch MR, Tsao GT, Narayan R, Rodwell W: 2,3-butanediol. In Comprehensive biotechnology. Volume 3. 1st edition. Edited by Murray M-Y. New York: Pergamon Press; 1985:932-947.

2. Ji XJ, Huang H, Ouyang PK: Microbial 2,3-butanediol production: a state-of -the-art review. Biotechnol Adv 2011, 29:351-364.

3. Celinska E, Grajek W: Biotechnological production of 2,3-butanediolcurrent state and prospects. Biotechnol Adv 2009, 27:715-725.

4. Zeng AP, Sabra W: Microbial production of diols as platform chemicals: recent progresses. Curr Opin Biotechnol 2011, 22:749-757.

5. Cho JH, Rathnasingh C, Song H, Chung BW, Lee HJ, Seung D: Fermentation and evaluation of Klebsiella pneumoniae and $K$. oxytoca on the production of 2,3-butanediol. Bioprocess Biosyst Eng 2012, 35:1081-1088.

6. Qureshi N, Cheryan M: Production of 2,3-butanediol by Klebsiella oxytoca. Appl Microbiol Biotechnol 1989, 30:440-443.

7. Ji XJ, Nie ZK, Huang H, Ren LJ, Peng C, Ouyang PK: Elimination of carbon catabolite repression in Klebsiella oxytoca for efficient 2,3-butanediol production from glucose-xylose mixtures. Appl Microbiol Biotechnol 2011, 89:1119-1125

8. Ji XJ, Huang H, Li S, Du J, Lian M: Enhanced 2,3-butanediol production by altering the mixed acid fermentation pathway in Klebsiella oxytoca. Biotechnol Lett 2008, 30:731-734.

9. Ji XJ, Huang H, Zhu JG, Ren LJ, Nie ZK, Du J, Li S: Engineering Klebsiella oxytoca for efficient 2, 3-butanediol production through insertional inactivation of acetaldehyde dehydrogenase gene. Appl Microbiol Biotechnol 2010, 85:1751-1758.

10. Ji XJ, Huang H, Du J, Zhu JG, Ren L, Hu N, Li S: Enhanced 2,3-butanediol production by Klebsiella oxytoca using a two-stage agitation speed control strategy. Bioresour Technol 2009, 100:3410-3414.

11. Shin SH, Kim S, Kim JY, Lee S, Um Y, Oh MK, Kim YR, Lee J, Yang KS: Complete genome sequence of Klebsiella oxytoca KCTC 1686, used in production of 2,3-butanediol. J Bacterio/ 2012, 194:2371-2372.

12. Liao TL, Lin AC, Chen E, Huang TW, Liu YM, Chang YH, Lai JF, Lauderdale TL, Wang JT, Chang SC, et al: Complete genome sequence of Klebsiella oxytoca E718, a New Delhi metallo-beta-lactamase-1-producing nosocomial strain. J Bacteriol 2012, 194:5454.

13. Yang G, Tian J, Li J: Fermentation of 1,3-propanediol by a lactate deficient mutant of Klebsiella oxytoca under microaerobic conditions. Appl Microbiol Biotechnol 2007, 73:1017-1024.

14. Champluvier B, Decallonne J, Rouxhet PG: Influence of sugar source (lactose, glucose, galactose) on 2,3-butanediol production by Klebsiella oxytoca NRRL-B199. Arch Microbiol 1989, 152:411-414. 
15. Jansen NB, Flickinger MC, Tsao GT: Production of 2,3-butanediol from Dxylose by Klebsiella oxytoca ATCC 8724. Biotechnol Bioeng 1984, 26:362-369.

16. Cao N, Xia Y, Gong CS, Tsao GT: Production of 2,3-butanediol from pretreated corn cob by Klebsiella oxytoca in the presence of fungal cellulase. Appl Biochem Biotechnol 1997, 63-65:129-139.

17. Homman T, Tag C, Biebl H, Deckwer WD, Schink B: Fermentation of glycerol to 1,3-propanediol by Klebsiella and Citrobacter strains. Appl Microbiol Biotechnol 1990, 33:121-126.

18. Ji XJ, Huang H, Du J, Zhu JG, Ren LJ, Li S, Nie ZK: Development of an industrial medium for economical 2,3-butanediol production through co-fermentation of glucose and xylose by Klebsiella oxytoca. Bioresour Technol 2009, 100:5214-5218

19. Nie ZK, Ji XJ, Huang H, Du J, Li ZY, Qu L, Zhang Q, Ouyang PK: An effective and simplified fed-batch strategy for improved 2,3-butanediol production by Klebsiella oxytoca. Appl Biochem Biotechnol 2011, 163:946-953.

20. Garg SK, Jain A: Fermentative production of 2, 3-butanediol: a review. Bioresour Technol 1995, 51:103-109.

21. Beronio PB Jr, Tsao GT: Optimization of 2,3-butanediol production by Klebsiella oxytoca through oxygen transfer rate control. Biotechnol Bioeng 1993, 42:1263-1269.

22. Beronio PB Jr, Tsao GT: An energetic model for oxygen-limited metabolism. Biotechnol Bioeng 1993, 42:1270-1276.

23. Qureshi N, Cheryan M: Effects of aeration on 2,3-butanediol production from glucose by Klebsiella oxytoca. J Ferment Bioeng 1989, 67:415-418.

24. Zhang G, Yang G, Wang X, Guo Q, Li Y, Li J: Influence of blocking of 2, 3-butanediol pathway on glycerol metabolism for 1,3-propanediol production by Klebsiella oxytoca. Appl Biochem Biotechnol 2012, 168:116-128.

25. Zhang Y, Huang Z, Du C, Li Y, Cao Z: Introduction of an NADH regeneration system into Klebsiella oxytoca leads to an enhanced oxidative and reductive metabolism of glycerol. Metab Eng 2009, 11:101-106.

26. Lee JW, Na D, Park JM, Lee J, Choi S, Lee SY: Systems metabolic engineering of microorganisms for natural and non-natural chemicals. Nat Chem Biol 2012, 8:536-546.

27. Lee JW, Kim TY, Jang YS, Choi S, Lee SY: Systems metabolic engineering for chemicals and materials. Trends Biotechnol 2011, 29:370-378.

28. Park JH, Lee SY: Towards systems metabolic engineering of microorganisms for amino acid production. Curr Opin Biotechnol 2008, 19:454-460.

29. Furusawa $C$, Horinouchi T, Hirasawa T, Shimizu H: Systems metabolic engineering: the creation of microbial cell factories by rational metabolic design and evolution. Adv Biochem Eng Biotechnol 2012. in press.

30. Becker J, Wittmann C: Systems and synthetic metabolic engineering for amino acid production - the heartbeat of industrial strain development. Curr Opin Biotechnol 2012, 23:718-726.

31. Becker J, Zelder O, Hafner S, Schroder H, Wittmann C: From zero to hero-design-based systems metabolic engineering of Corynebacterium glutamicum for L-lysine production. Metab Eng 2011, 13:159-168.

32. Kanehisa M, Goto S, Sato Y, Furumichi M, Tanabe M: KEGG for integration and interpretation of large-scale molecular data sets. Nucleic Acids Res 2012, 40:D109-D114.

33. Caspi R, Altman T, Dreher K, Fulcher CA, Subhraveti $P$, Keseler IM, Kothari A, Krummenacker M, Latendresse M, Mueller LA, et al: The MetaCyc database of metabolic pathways and enzymes and the BioCyc collection of pathway/genome databases. Nucleic Acids Res 2012, 40:D742-D753.

34. Blomqvist K, Nikkola M, Lehtovaara P, Suihko ML, Airaksinen U, Straby KB, Knowles JK, Penttila ME: Characterization of the genes of the 2,3butanediol operons from Klebsiella terrigena and Enterobacter aerogenes. J Bacteriol 1993, 175:1392-1404.

35. Park JM, Kim TY, Lee SY: Constraints-based genome-scale metabolic simulation for systems metabolic engineering. Biotechnol Adv 2009, 27:979-988.

36. Orth JD, Conrad TM, Na J, Lerman JA, Nam H, Feist AM, Palsson BO: A comprehensive genome-scale reconstruction of Escherichia coli metabolism-2011. Mol Syst Biol 2011, 7:535

37. Schuetz R, Kuepfer $L$, Sauer U: Systematic evaluation of objective functions for predicting intracellular fluxes in Escherichia coli. Mol Syst Biol 2007, 3:119.

38. Saier MH Jr, Tran CV, Barabote RD: TCDB: the transporter classification database for membrane transport protein analyses and information. Nucleic Acids Res 2006, 34:D181-D186.

39. Ren Q, Chen K, Paulsen IT: TransportDB: a comprehensive database resource for cytoplasmic membrane transport systems and outer membrane channels. Nucleic Acids Res 2007, 35:D274-D279.
40. Gombert AK, Nielsen J: Mathematical modelling of metabolism. Curr Opin Biotechnol 2000, 11:180-186.

41. Edwards JS, Ramakrishna R, Schilling CH, Palsson BO: Metabolic flux balance analysis. In Metabolic engineering. Edited by Lee SY, Papoutsakis ET. New York: Marcel Dekker Inc; 1999:13-57.

42. Stephanopoulos G, Aristidou AA, Nielsen JH: Metabolic engineering: principles and methodologies. San Diego: Academic; 1998

43. Varma A, Palsson BO: Metabolic flux balancing: basic concepts, scientific and practical use. Biotechnology 1994, 12:994-998.

44. Delgado J, Liao JC: Inverse flux analysis for reduction of acetate excretion in Escherichia coli. Biotechnol Prog 1997, 13:361-367.

45. Lee KH, Park JH, Kim TY, Kim HU, Lee SY: Systems metabolic engineering of Escherichia coli for L-threonine production. Mol Syst Biol 2007, 3:149.

46. Jung YK, Kim TY, Park SJ, Lee SY: Metabolic engineering of Escherichia coli for the production of polylactic acid and its copolymers. Biotechnol Bioeng 2010, 105:161-171.

doi:10.1186/1475-2859-12-20

Cite this article as: Park et al:: Genome-scale reconstruction and in silico analysis of Klebsiella oxytoca for 2,3-butanediol production. Microbial Cell Factories 2013 12:20.

\section{Submit your next manuscript to BioMed Central and take full advantage of:}

- Convenient online submission

- Thorough peer review

- No space constraints or color figure charges

- Immediate publication on acceptance

- Inclusion in PubMed, CAS, Scopus and Google Scholar

- Research which is freely available for redistribution
C) Biomed Central 